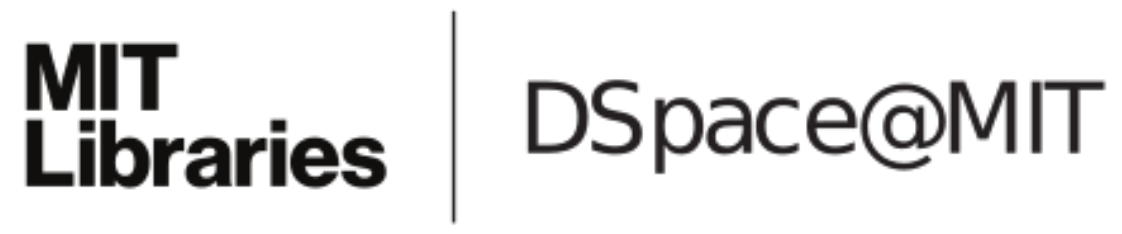

\author{
MIT Open Access Articles
}

Resource Competition Shapes the Response of Genetic Circuits

The MIT Faculty has made this article openly available. Please share how this access benefits you. Your story matters.

Citation: Qian, Yili et al. "Resource Competition Shapes the Response of Genetic Circuits." ACS Synthetic Biology 6, 7 (April 2017): 1263-1272 @ 2017 American Chemical Society

As Published: http://dx.doi.org/10.1021/ACSSYNBI0.6B00361

Publisher: American Chemical Society (ACS)

Persistent URL: http://hdl.handle.net/1721.1/119015

Version: Author's final manuscript: final author's manuscript post peer review, without publisher's formatting or copy editing

Terms of Use: Article is made available in accordance with the publisher's policy and may be subject to US copyright law. Please refer to the publisher's site for terms of use. 


\title{
Resource Competition Shapes the Response of Genetic Circuits
}

\author{
Yili Qian ${ }^{1 \dagger}$, Hsin-Ho Huang ${ }^{1 \dagger}$, José I. Jiménez ${ }^{1,2}$, Domitilla Del Vecchio ${ }^{1,3, *}$
}

${ }^{1}$ Department of Mechanical Engineering, Massachusetts Institute of Technology, Cambridge, MA, USA

${ }^{2}$ Faculty of Health of Medical Sciences, University of Surrey, Guildford, UK

${ }^{3}$ Synthetic Biology Center, Massachusetts Institute of Technology, Cambridge, MA, USA

$\dagger$ These authors contributed equally to this work.

* Correspondence: ddv@mit.edu

\begin{abstract}
A common approach to design genetic circuits is to compose gene expression cassettes together. While appealing, this modular approach is challenged by the fact that expression of each gene depends on the availability of transcriptional/translational resources, which is in turn determined by the presence of other genes in the circuit. This raises the question of how competition for resources by different genes affects a circuit's behavior. Here, we create a library of genetic activation cascades, where we explicitly tune the resource demand by each gene. We develop a general Hill-function-based model that incorporates resource competition effects through resource demand coefficients. These coefficients lead to non-regulatory interactions among genes that reshape circuit's behavior. For the activation cascade, such interactions result in surprising biphasic or monotonically decreasing responses. Finally, we use resource demand coefficients to guide the choice of ribosome binding site (RBS) and DNA copy number to restore the cascade's intended monotonically increasing response. Our results demonstrate how unintended circuit's behavior arises from resource competition and provide a model-guided methodology to minimize the resulting effects.
\end{abstract}

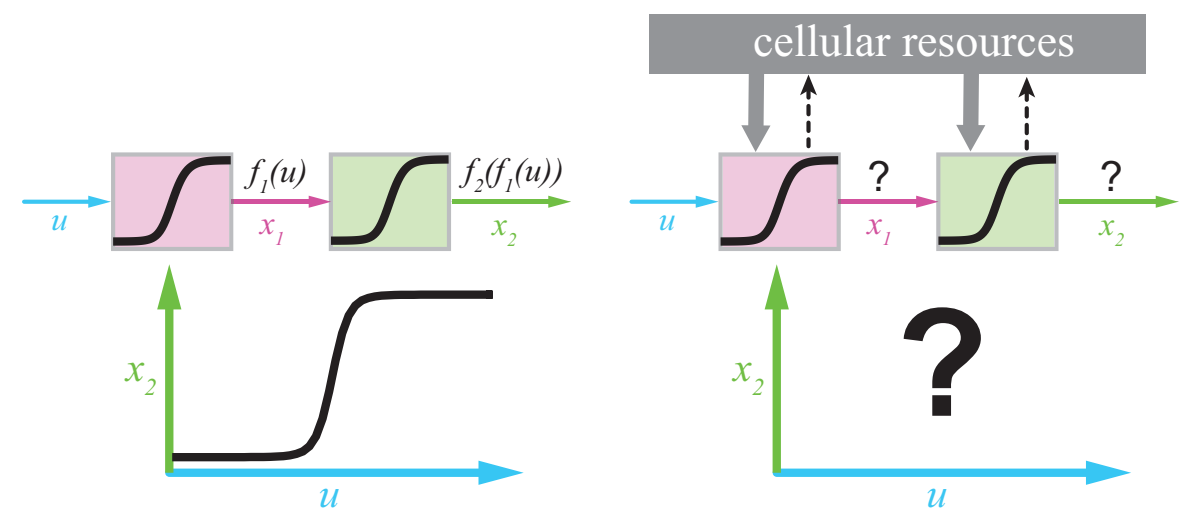

\section{Introduction}

Predicting the behavior of genetic circuits in living cells is a recurring challenge in synthetic biology [1]. Genetic circuits are often viewed as interconnections of gene expression cassettes, which we call nodes. Each cassette (node) is composed of core gene expression processes, that are, transcription and translation. Here, we view each node as the input/output system that takes transcription factors (TFs) as inputs, which regulate the expression of an output TF. Although in an ideal scenario we 
would like to predict the behavior of a circuit from that of its composing nodes characterized in isolation, in reality, a node's behavior often depends on its context, including other nodes in the same circuit and the host cell environment [2]. This fact significantly limits our current ability to design genetic circuits that behave as intended. There are a number of causes to context dependence, including unknown structural interactions between adjacent genetic sequences [3], loading of TFs by target DNA sites (retroactivity) $[4,5,6]$, unintended coupling between synthetic genes and host cell growth (host-circuit interaction) [7,8,9], and competition among synthetic genes with each other for common transcriptional and translational resources [10, 11, 12, 13, 14]. Context dependence due to structural interactions and retroactivity has been addressed by engineering insulation parts and devices $[15,16,6,17,18]$ and that due to host-circuit interaction may be mitigated to some extent by orthogonal RNA polymerases (RNAPs) and ribosomes [19, 20, 21, 22]. By contrast, the characterization and mitigation of competition for shared resources among synthetic genes remain largely unexplored.

Expression of all genes in a genetic circuit relies on a common pool of transcriptional and translational resources. In particular, the availability of RNAPs and ribosomes has been identified as a major bottleneck for gene expression in bacteria $[23,24,25,26]$. When a node is activated, it depletes the pool of free RNAPs and ribosomes, reducing their availability to other nodes in the circuit. This can potentially affect the behavior of a circuit altogether. Recent experimental results have demonstrated that competition for transcriptional and translational resources, chiefly ribosomes, can couple the expression of two synthetic genes that are otherwise unconnected [10, 12]. These works demonstrate that upon induction of a synthetic gene, the expression level of a constitutively expressed gene on the same plasmid can be reduced by more than $60 \%$. Similar trade-offs have been observed in cell-free systems [27] and in computational models [11, 13, 14, 28].

In this paper, we seek to determine how competition for resources by the genes constituting a synthetic genetic circuit changes the intended circuit's behavior. To address this question, we perform a combined modeling and experimental study. In particular, we develop a general mathematical model that explicitly includes competition for RNAPs and ribosomes in Hill-function models of gene expression. In our models, resource demand coefficients quantify the demand for resources by each node and shape the emergent input/output response of a genetic circuit. We construct a library of synthetic genetic activation cascades in which we tune the resource demand coefficients by changing the RBS strength of the cascade's genes and DNA copy number. When the resource demand coefficients are large, the dose response curve of the cascade can either be biphasic or monotonically decreasing. When we decrease the resource demand coefficients, we restore the intended cascade's monotonically increasing dose response curve. For general circuits, of our model reveals that due to non-zero resource demand coefficients, resource competition gives rise to non-regulatory interactions among nodes. We give a general rule for drawing the effective interaction graph of any genetic circuit that combines both intended and unintended interactions.

\section{Results}

\section{Surprising biphasic response results from a cascade of two activators}

We built a two-stage activation cascade as follows. Node 1 uses transcriptional activation by the effector $N$-hexanoyl-L-homoserine lactone (AHL), the LuxR protein and the lux promoter (Figure 1A). The LuxR protein is constitutively expressed by the lac promoter in a LacI-deficient host strain. To characterize the dose response curve of this node, we placed red fluorescent protein (RFP) under the control of the lux promoter. An increase in AHL concentration increases the holo-form of ligand-responsive TF (i.e., LuxR+AHL complex), promoting production of RFP (Figure 1A).

Node 2 employs transcriptional activation by the effector salicylate (SAL), the NahR protein, and the sal promoter to express green fluorescent protein (GFP) as fluorescence output. NahR production is under the control of the lux promoter. We applied a constant amount of AHL (100 nM) and expressed LuxR constitutively to produce a constant supply of NahR. By increasing the amount of $\mathrm{SAL}$, the holo-form of ligand-responsive TF (NahR+SAL complex) increases, activating production of GFP. We confirmed that GFP concentration increases monotonically with SAL input (Figure 1B). 


\section{A First activation stage}
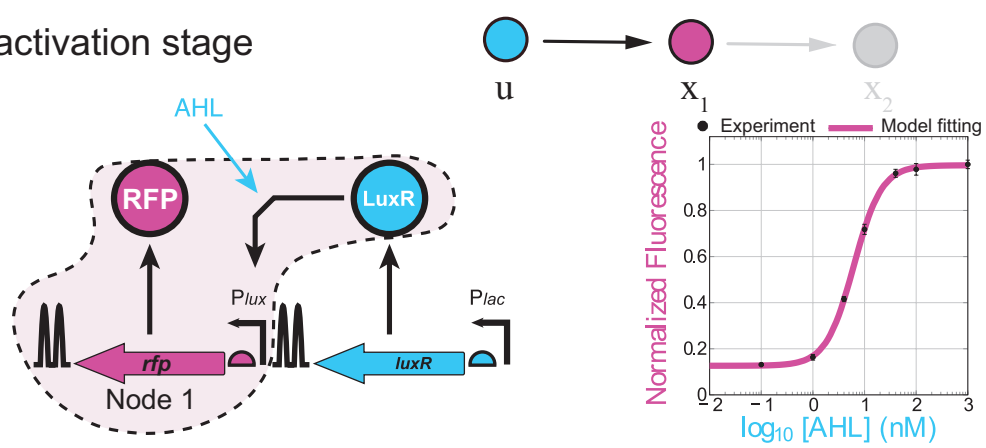

\section{B Second activation stage}
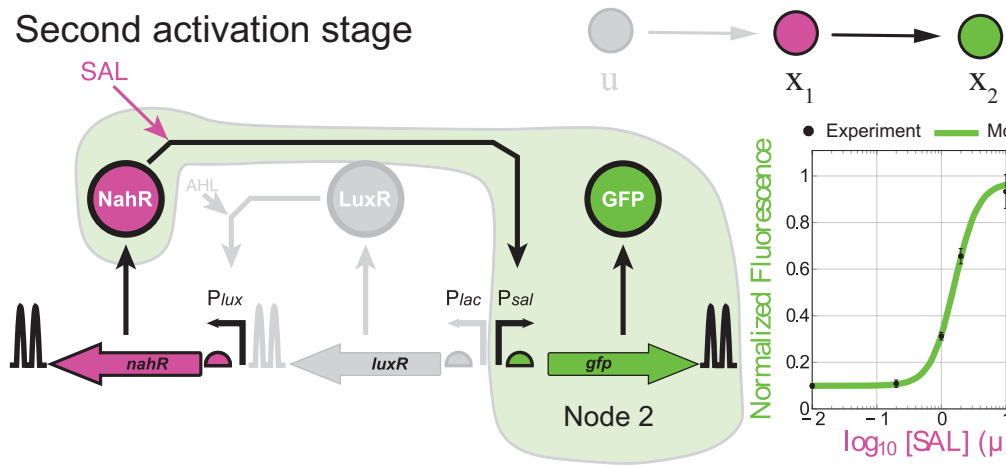

- Experiment Model fitting

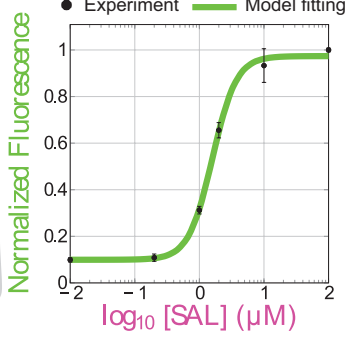

C Two-stage activation cascade
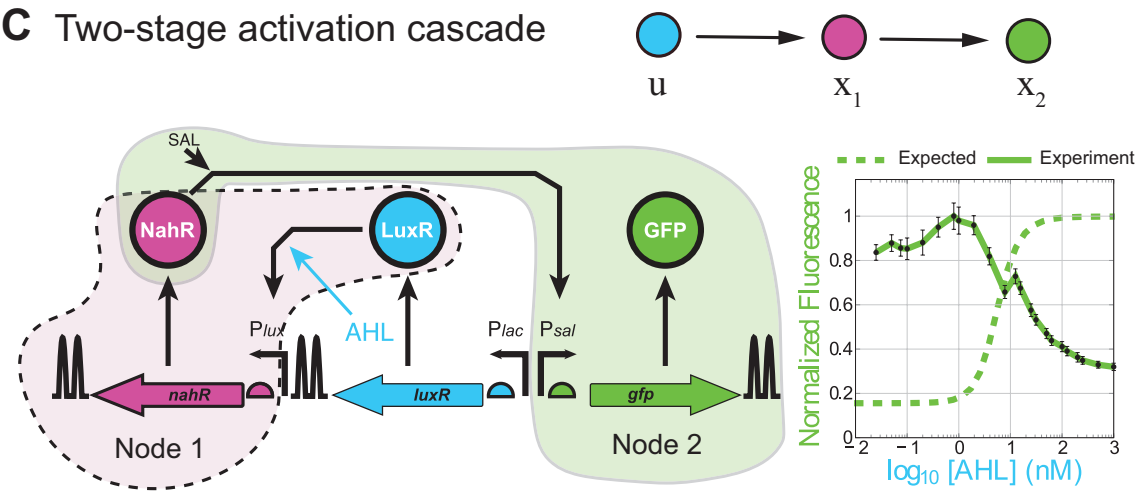

Figure 1: Failure of modular composition in a simple two-stage activation cascade. (A) The first activation stage consists of a node that takes as input the transcription activator LuxR to promote production of RFP as output in the presence of AHL, resulting in a monotonically increasing dose response curve. Upward arrows with leftward/rightward tips represent promoters, semicircles represent RBS, and double hairpins represent terminators. (B) The second activation stage consists of a node that takes as input the transcription activator NahR to promote production of GFP as output in the presence of SAL, resulting in a monotonically increasing dose response curve. (C) The two-stage activation cascade CAS 1/30 was built by connecting the two nodes in a cascade topology. Biphasic dose response curve (solid line) of the cascade was observed instead of the expected monotonically increasing dose response curve (dashed line), which is the composition of the two increasing Hill functions for the individual nodes according to equation (3). All experimental data represent mean values and standard deviations of populations in the steady state analyzed by flow cytometry in three independent experiments. Each plot is normalized to its maximum fluorescence value (see SI Section A6 for details). 
To build a two-stage activation cascade (CAS 1/30), we connected the two nodes by replacing the RFP in node 1 by NahR. With a saturating amount of SAL $(1 \mathrm{mM})$, NahR protein can be regarded as the output of node 1 and the input to node 2. Therefore, increased AHL concentration leads to increased NahR concentration, and hence to increased concentration of NahR+SAL complex, resulting in increased production of GFP (cascade output).

The expected behavior of this cascade is a monotonically increasing GFP fluorescence as AHL is increased. This can be easily predicted by a standard Hill-function model of the circuit. Specifically, letting $\mathrm{u}$ denote LuxR+AHL complex, $\mathrm{x}_{1}$ the TF NahR, and $\mathrm{x}_{2}$ the GFP output, and using $u$, $x_{1}$ and $x_{2}$ (italics) to represent their concentrations, we obtain the following ordinary differential equation (ODE) model (see SI Section B1 for details):

$$
\frac{\mathrm{d} x_{1}}{\mathrm{~d} t}=\underbrace{T_{1} F_{1}(u)}_{\text {node } 1 \text { expression }}-\underbrace{\gamma_{1} x_{1}}_{\text {decay }}, \quad \frac{\mathrm{d} x_{2}}{\mathrm{~d} t}=\underbrace{T_{2} F_{2}\left(x_{1}\right)}_{\text {node } 2 \text { expression }}-\underbrace{\gamma_{2} x_{2}}_{\text {decay }},
$$

where $T_{i}(i=1,2)$ are lumped parameters describing maximal expression rate of the nodes (defined in equation (S13) in SI), $\gamma_{i}$ are protein decay rate constants, and $F_{1}(u)$ and $F_{2}\left(x_{1}\right)$ are standard increasing Hill-functions [29, 30] whose maxima are normalized to 1:

$$
F_{1}(u)=\frac{\beta_{1}+\left(u / k_{1}\right)^{n_{1}}}{1+\left(u / k_{1}\right)^{n_{1}}}, \quad F_{2}\left(x_{1}\right)=\frac{\beta_{2}+\left(x_{1} / k_{2}\right)^{n_{2}}}{1+\left(x_{1} / k_{2}\right)^{n_{2}}} .
$$

In equations $(2), k_{i}$ are the dissociation constants between the activators, $\mathrm{u}$ and $\mathrm{x}_{1}$, and their respective DNA targets. Dimensionless parameters $\beta_{i}<1$ characterize basal expressions, and $n_{i}$ are Hill coefficients capturing cooperativities of the TF and promoter bindings. Setting the time derivatives to zero, we obtain the dose response of the cascade as the composition of two increasing Hill-functions:

$$
x_{2}=\frac{T_{2}}{\gamma_{2}} F_{2}\left(\frac{T_{1}}{\gamma_{1}} F_{1}(u)\right) .
$$

It is clear from (3), that independent of parameters, the steady state of $x_{2}$ (GFP concentration) always increases with $u$ (AHL concentration). Surprisingly, the experimental results contradict this rather trivial prediction. In fact, although the input/output response of both composing nodes are monotonically increasing (Figure 1A-B), their cascade shows a biphasic dose response curve, in which the GFP fluorescence decreases with increased concentrations of AHL for high AHL concentrations (Figure 1C). This fact clearly demonstrates that while the standard model well represents the activation behavior of each individual node, its predictive ability is lost when the two nodes are connected and thus are simultaneously activated.

\section{A cascade model taking into account resource competition predicts non- regulatory interactions}

An underlying assumption in the standard Hill-function model (1) is that the concentrations of free RNAPs and ribosomes can be regarded as constant parameters [29, 30] (refer to SI Section $\mathrm{B} 1)$. In reality, because the concentration of these resources is limited [23, 24, 26, 31], their free concentration should depend on the extent to which different nodes in a circuit demand them. With reference to Figure $1 \mathrm{C}$, the biphasic response of $x_{2}$ can be explained by the following resource competition mechanism. When we increase $u$, node 1 sequesters more RNAPs and ribosomes to produce $\mathrm{x}_{1}$, decreasing the amount of free resources, which in turn result in decreased transcription and translation rates at node 2 .

We therefore created a model that explicitly accounts for the limited concentration of RNAPs and ribosomes and for their competition by the two cascade genes. For a given growth rate, the total concentrations of RNAPs and ribosomes can be assumed constant parameters [9, 23]. Considering the conservation law for these resources and solving for their free concentrations (see SI Section B2), 
we obtain the following modified Hill-function model:

$$
\frac{\mathrm{d} x_{1}}{\mathrm{~d} t}=\underbrace{\frac{T_{1} F_{1}(u)}{1+J_{1} F_{1}(u)+J_{2} F_{2}\left(x_{1}\right)}}_{G_{1}\left(u, x_{1}\right): \text { node } 1 \text { expression }}-\underbrace{\gamma_{1} x_{1}}_{\text {decay }}, \quad \frac{\mathrm{d} x_{2}}{\mathrm{~d} t}=\underbrace{\frac{T_{2} F_{2}\left(x_{1}\right)}{1+J_{1} F_{1}(u)+J_{2} F_{2}\left(x_{1}\right)}}_{G_{2}\left(u, x_{1}\right): \text { node } 2 \text { expression }}-\underbrace{\gamma_{2} x_{2}}_{\text {decay }} .
$$

in which $F_{1}(u)$ and $F_{2}\left(x_{1}\right)$ are defined in equations (2). The lumped dimensionless parameters $J_{i}$ $(i=1,2)$ can be understood as an indicator of maximal resource demand by node $\mathrm{i}$, and we call them resource demand coefficients. They are defined as:

$$
J_{i}:=\frac{p_{i, T}}{K_{i}} \cdot\left(1+\frac{\alpha_{i}}{\kappa_{i} \delta_{i}} y_{T}\right), i=1,2,
$$

where $p_{i, T}$ is the DNA copy number of node $\mathrm{i} ; \alpha_{i}$ is its transcription elongation rate constant, describing the average number of mRNAs transcribed from a single DNA molecule in unit time; $\delta_{i}$ is mRNA decay rate constant, and $y_{T}$ is the total concentration of RNAPs. The ability of each DNA molecule (mRNA molecule) to occupy free RNAPs (ribosomes) is characterized by lumped coefficient $K_{i}\left(\kappa_{i}\right)$, defined in equations (S3) and (S8) in SI. They can be viewed as effective dissociation constants that decrease with (i) stronger affinity between activated promoter (RBS) in node i and free RNAPs (ribosomes), and (ii) slower transcription (translation) elongation rate constants. Physically, resource demand coefficient of node i $\left(J_{i}\right)$ increases as (I) the total number of promoter sites $\left(p_{i, T}\right)$ increases, (II) the total number of mRNA molecules $\left(p_{i, T} \alpha_{i} y_{T} / K_{i} \delta_{i}\right)$ increases, (III) the ability of each DNA molecule to sequester free RNAPs $\left(1 / K_{i}\right)$ increases, or (IV) the ability of each mRNA molecule to sequester free ribosomes $\left(1 / \kappa_{i}\right)$ increases. When transcriptional activation is applied, the portion of resources allocated to each node is quantified by $J_{1} F_{1}(u)$ and $J_{2} F_{2}\left(x_{1}\right)$, respectively, and follow the conservation law (see SI Section B5 for derivation):

$$
\underbrace{y_{T} \cdot z_{T}}_{\text {total available resources }}=\underbrace{y \cdot z}_{\text {free resources }}+\underbrace{y \cdot z \cdot J_{1} F_{1}(u)}_{\text {resource demand of node } 1}+\underbrace{y \cdot z \cdot J_{2} F_{2}\left(x_{1}(u)\right)}_{\text {resource demand of node } 2},
$$

where $y_{T}(y)$ and $z_{T}(z)$ are the total (free) amount of RNAPs and ribosomes, respectively.

The major difference between model (4) and the standard Hill-function model (1) is the common denominator $1+J_{1} F_{1}(u)+J_{2} F_{2}\left(x_{1}\right)$ in effective gene expression rates $G_{1}\left(u, x_{1}\right)$ and $G_{2}\left(u, x_{1}\right)$. In a resource-abundant situation where RNAPs and ribosomes bound to both nodes are much smaller than their free amounts $\left(y \approx y_{T}\right.$ and $\left.z \approx z_{T}\right)$, we must have $J_{1}, J_{2} \ll 1$, and model (4) reduces to the standard Hill-function model (1). Detailed proof of this result is in SI Section B5. Because of this common denominator in $G_{1}\left(u, x_{1}\right)$ and $G_{2}\left(u, x_{1}\right)$ in equation (4), the production of each node depends on all TFs present in the circuit as opposed to depending only on its own TF inputs as in equation (1). In particular, regardless of parameters, we always have the following effective interactions among the cascade nodes (see SI Section B3.1 for derivation):

$$
\frac{\partial G_{1}}{\partial u}>0 \Rightarrow \mathrm{u} \rightarrow \mathrm{x}_{1}, \quad \frac{\partial G_{2}}{\partial u}<0 \Rightarrow \mathrm{u} \dashv \mathrm{x}_{2}, \quad \frac{\partial G_{1}}{\partial x_{1}}<0 \Rightarrow \mathrm{x}_{1} \dashv \mathrm{x}_{1}, \quad \frac{\partial G_{2}}{\partial x_{1}}>0 \Rightarrow \mathrm{x}_{1} \rightarrow \mathrm{x}_{2} .
$$

While interactions $\mathrm{u} \rightarrow \mathrm{x}_{1}$ and $\mathrm{x}_{1} \rightarrow \mathrm{x}_{2}$ are due to the intended transcriptional activations, the other two interactions, $u \dashv x_{2}$ and $x_{1} \dashv x_{1}$ are not present in the standard model (1). They can be regarded as non-regulatory interactions arising from resource competition among nodes. In particular, the non-regulatory interaction $\mathrm{u} \dashv \mathrm{x}_{2}$ is due to the fact that as $u$ increases, production of $\mathrm{x}_{1}$ is activated, depleting the pool of free resources, thus reducing the amount available to initiate transcription and translation of $\mathrm{x}_{2}$. Similarly, increase in $x_{1}$ activates production of $\mathrm{x}_{2}$, reducing resources available to its own expression to create the self repression loop $\mathrm{x}_{1} \dashv \mathrm{x}_{1}$.

Based on (7), the effective interactions among nodes in an activation cascade are shown in Figure $2 \mathrm{~A}$, where black solid lines represent intended regulatory interactions and red dashed lines represent non-regulatory interactions due to resource competition. The non-regulatory interactions change the topology of this activation cascade into a type 3 incoherent feed-forward loop (IFFL) [29], where $\mathrm{x}_{2}$ production is jointly affected by regulatory activation from $\mathrm{x}_{1}$ and non-regulatory repression from u. It is well-known that, depending on parameters, the dose response curve of an IFFL can be 


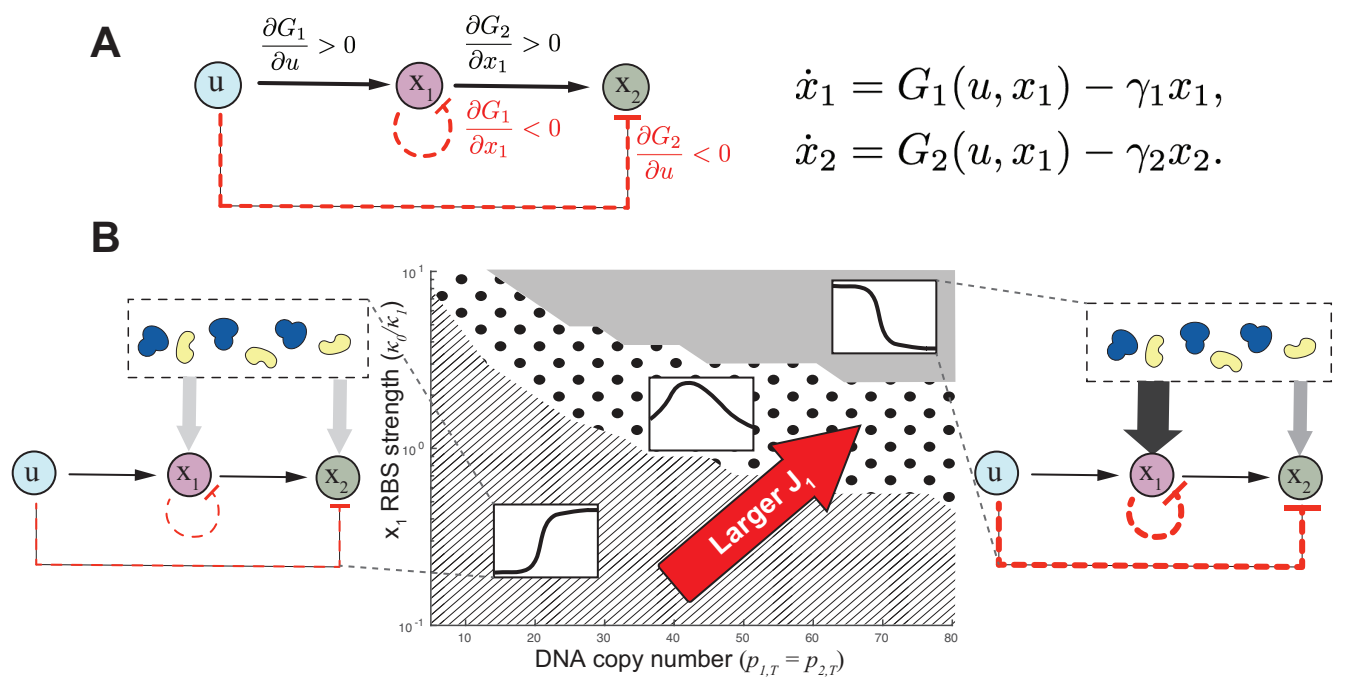

Figure 2: Activation cascade becomes a type 3 IFFL due to resource competition. (A) Effective interactions among nodes in a two-stage activation cascade with resource competition. Black solid lines are regulatory interactions, and red dashed lines represent non-regulatory interactions due to resource competition. (B) Parameter space illustrating diverse cascade dose response curves obtained from numerical simulation when the resource demand coefficient $J_{1}$ is changed. The horizontal axis shows the DNA copy number, and the vertical axis shows the RBS strength of node 1 . Numerical values on the vertical axis represent the ratio between a baseline dissociation constant $\kappa_{0}$ between RBS and ribosomes $(15 \mu \mathrm{M})$, and that of node $1\left(\kappa_{1}\right)$. The cascade has monotonically decreasing, biphasic or monotonically increasing dose response curve depending on whether the parameters fall into the gray, dotted and grid shaded region in the parameter space, respectively. Chemical reactions are described in SI Section B1 and B2, parameters and detailed simulation data are in SI Section B10.

monotonically increasing, decreasing or biphasic [32,33]. If transcriptional activation $\mathrm{u} \rightarrow \mathrm{x}_{1} \rightarrow \mathrm{x}_{2}$ is stronger than non-regulatory repression $u \dashv x_{2}$, then the dose response curve is monotonically increasing. Conversely, if the non-regulatory repression is stronger than transcriptional activation, the dose response curve becomes monotonically decreasing. Biphasic responses can be observed when transcriptional activation dominates at lower $u$ level, and resource-competition-induced nonregulatory repression becomes more significant at higher $u$. A detailed analytical treatment of this reasoning is in SI Section B3.1.

The strength of the non-regulatory repression $\mathrm{u} \dashv \mathrm{x}_{2}$ can be reduced by decreasing resource demand coefficient of node $1\left(J_{1}\right)$. In particular, as a result, the dose response curve of an activation cascade is monotonically increasing when $J_{1} \ll 1$ (see SI Section B3.2). Conversely, we expect the dose response curve to be monotonically decreasing when $J_{1}$ is large, and to be biphasic for intermediate values of $J_{1}$. Based on the definition of resource demand coefficient in (5), we can decrease $J_{1}$ by choosing weak node 1 RBS strength and low DNA copy number. We simulated the dose response curves of activation cascades with different node 1 RBS strengths and DNA copy numbers, presented in the parameter space in Figure 2B. The lower left corner of the parameter space corresponds to the cascade with the smallest $J_{1}$, and the upper right corner corresponds to the largest $J_{1}$. In accordance with these predictions, simulation in Figure $2 \mathrm{~B}$ confirms that smaller $J_{1}$ (weak $\mathrm{x}_{1}$ RBS and low DNA copy number) results in monotonically increasing response (grid shaded region), while larger $J_{1}$ (strong $\mathrm{x}_{1}$ RBS and high DNA copy number) results in monotonically decreasing response (gray region). The dotted region corresponds to intermediate values of $J_{1}$ which result in biphasic response. 


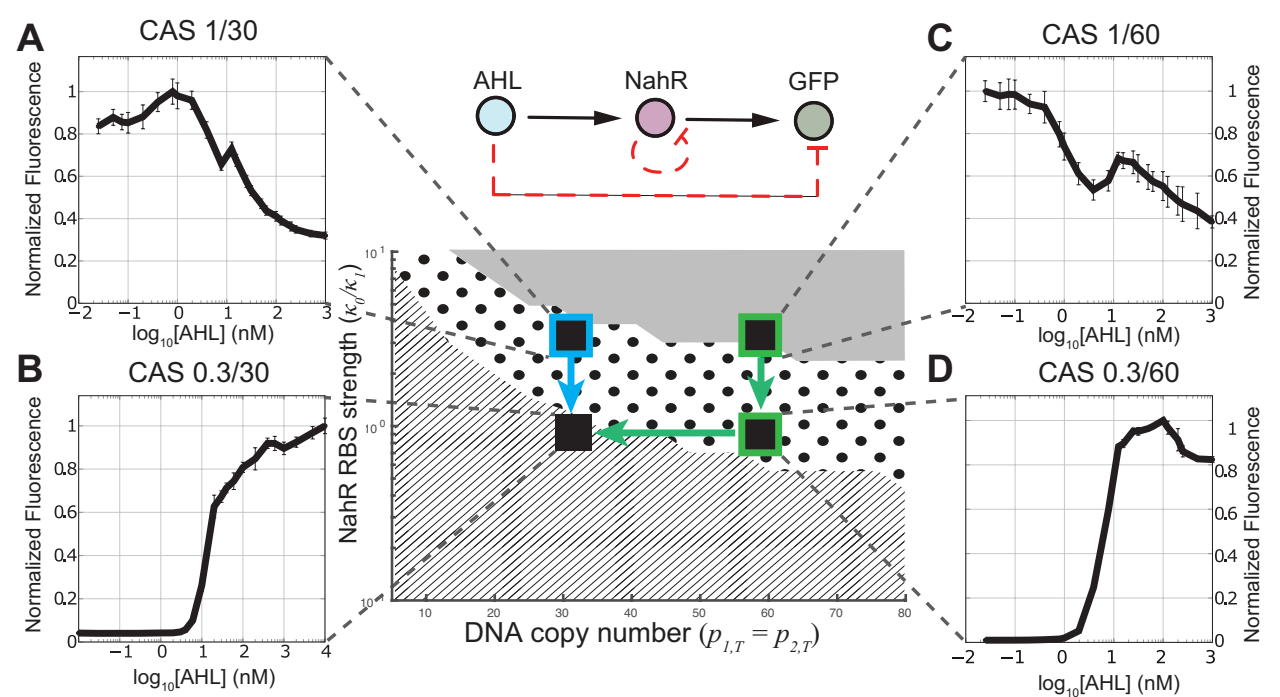

Figure 3: A monotonically increasing dose response curve can be restored by tuning DNA copy number and NahR RBS strength. The dose response curves of circuits CAS 1/30 (A) and CAS 1/60 (C) were biphasic and monotonically decreasing, respectively. By reducing the RBS strength of NahR, the dose response of CAS 0.3/30 (B) became monotonically increasing, and that of CAS 0.3/60 (D) becomes biphasic. Further decreasing the copy number of CAS 0.3/60 to CAS 0.3/30 restored the monotonically increasing dose response curve. Experimental results are presented on top of the parameter space created in Figure 2 by simulation. Blue and green arrows represent design actions to restore the monotonically increasing dose response curves starting from failed cascades CAS $1 / 30$ and CAS $1 / 60$, respectively. Mean values and standard deviations of fluorescence intensities at the steady state are calculated from three independent experiments analyzed by flow cytometry and normalized to the maximum value in each plot (see SI Section A6).

\section{Model-guided design recovers monotonically increasing response of the cascade}

Based on the simulation map in Figure 2 and the mathematical analysis of model (4) described in the previous section, we created a library of activation cascades in which each cascade should result into one of the three different behaviors shown in Figure 2. This library is composed of cascades that differ in the value of the resource demand coefficient of NahR $\left(J_{1}\right)$, with the rationale that we can mitigate the strength of non-regulatory interaction $\mathrm{u} \dashv \mathrm{x}_{2}$ to recover the intended monotonically increasing dose response curve of the cascade. In particular, starting from CAS $1 / 30$, whose dose response curve is biphasic (Figure 3A), we designed circuit CAS $0.3 / 30$ with about $30 \%$ RBS strength [12] of NahR compared to CAS $1 / 30$, theoretically resulting in a reduction of $J_{1}$. We therefore expect a reduction of the $\mathrm{u} \dashv \mathrm{x}_{2}$ interaction strength, leading to a monotonically increasing dose response curve, which is confirmed by the experiment (Figure 3B).

Similarly, we constructed another cascade circuit CAS 1/60 in which the DNA copy number is about twice as that of CAS 1/30 (about 60 vs 30). According to our model, resource demand coefficient of NahR $J_{1}$ in CAS $1 / 60$ should double compared to that of circuit CAS $1 / 30$. Therefore, we expect a possibly monotonically decreasing dose response curve. Experiments confirm this prediction (Figure 3C). A local increase in GFP fluorescence at about $10 \mathrm{nM}$ AHL is due to the two-step multimerization of NahR proteins [34], which is detailed in SI Section A5. To obtain a monotonically increasing dose response curve from this circuit, we first reduced NahR resource demand coefficient $J_{1}$ by designing a circuit CAS $0.3 / 60$, whose NahR RBS strength is $30 \%$ compared to that of CAS $1 / 60$. Theoretically, depending on parameters, reduced $J_{1}$ can lead to either monotonically increasing or biphasic dose response curves (see Figure 2B). Our experiment show that the response of CAS $0.3 / 60$ is indeed biphasic (Figure 3D). To restore a monotonically increasing dose response curve, we can further decrease $J_{1}$ by reducing DNA copy number to create circuit CAS $0.3 / 30$, whose dose response curve is monotonically increasing (Figure 3B). 


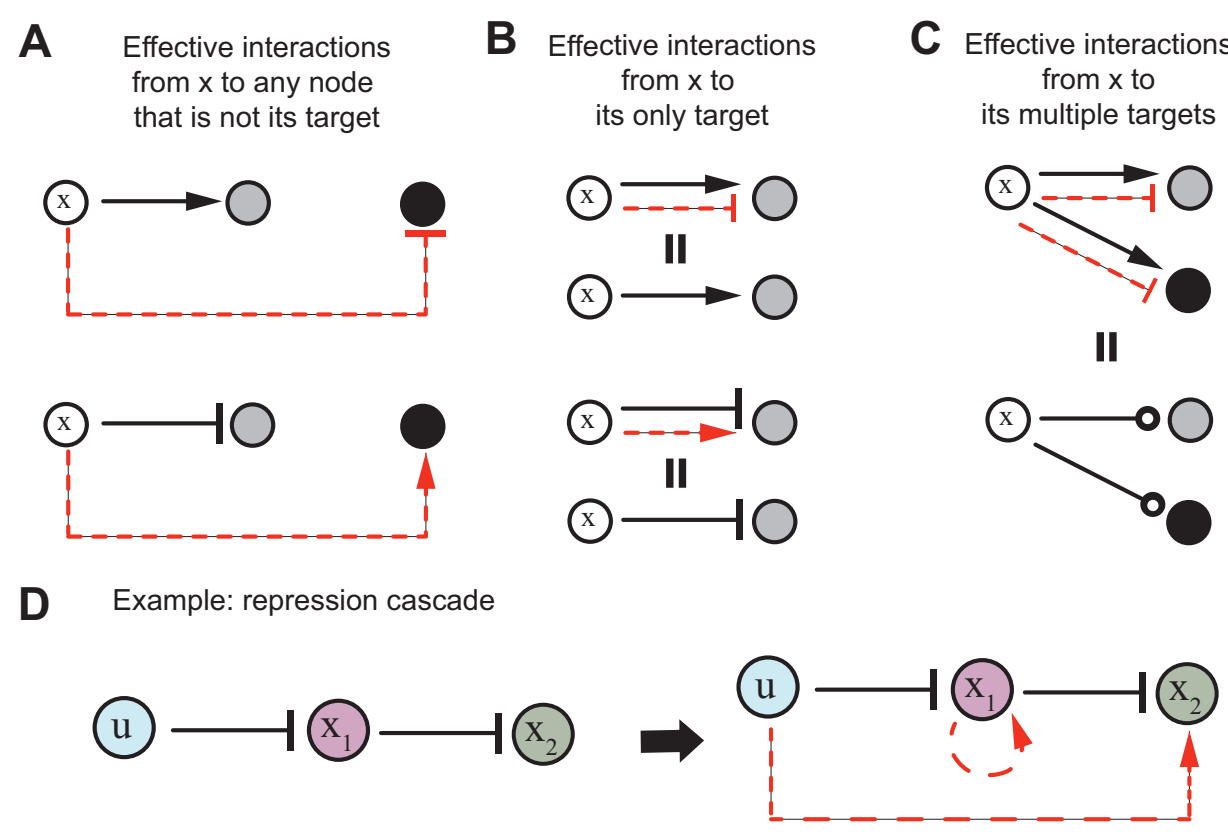

Figure 4: Rules to determine effective interaction graphs arising from resource competition in any genetic circuit. (A) If node $\mathrm{x}$ is an activator (repressor), then it is an effective repressor (activator) for all nodes that are not its target. (B) If node $\mathrm{x}$ has only one target, then resource competition does not change the nature of interaction from $\mathrm{x}$ to its target. (C) If node $\mathrm{x}$ regulates multiple targets, then the effective interaction from $\mathrm{x}$ to its targets are undetermined. (D) Applying the rules in A-C, a repression cascade becomes a type 4 coherent feed-forward loop due to resource competition.

\section{General rules to draw effective interactions in genetic circuits}

Interaction graphs, which use directed edges to represent regulatory interactions, are a convenient graphical tool to design and/or analyze the qualitative behavior of a genetic circuit [29]. Here, we expand the concept of interaction graph to incorporate non-regulatory interactions due to resource competition. We call the resultant interaction graph an effective interaction graph, which includes both regulatory interactions and non-regulatory interactions due to resource competition. In an effective interaction graph, we draw $\mathrm{x} \rightarrow \mathrm{y}(\mathrm{x} \dashv \mathrm{y})$ if increased amount of TF $\mathrm{x}$ promotes (inhibits) production of protein $\mathrm{y}$. We draw $\mathrm{x} \multimap \mathrm{y}$ if the effect of $\mathrm{x}$ on $\mathrm{y}$ depends on parameters and/or $\mathrm{x}$ concentration, and is thus undetermined in general.

The resource competition model (4) and effective interactions identified in Figure 2A for the activation cascade can be generalized to study genetic circuits with arbitrary topology in resource limited environments. In SI Section B4, we derive a general model (see equation (S55)) to determine effective interactions in any genetic circuit with resource competition. The essentials of this general model can be visualized by a set of graphical rules based on known regulatory interactions. These graphical rules are summarized in Figure 4A-C, in which we use black solid lines to represent regulatory interactions and red dashed lines to represent non-regulatory interactions arising from resource competition.

In summary, if a TF $\mathrm{x}$ is transcriptionally activating (repressing) a gene, which we call its target, then $\mathrm{x}$ is repressing (activating) all other genes that are not its target in a non-regulatory fashion (Figure 4A). This behavior stems from the fact that when $\mathrm{x}$ is a transcription activator (repressor), it promotes (inhibits) expression of its target, resulting it to sequester (release) resources, and consequently reducing (increasing) the amount of resources available to other nodes in the circuit. When $\mathrm{x}$ regulates only one target, while the strength of the effective interaction from $\mathrm{x}$ to its target is affected by resource competition, its nature (i.e. activation vs. repression) remains the same as the regulatory interaction (Figure 4B). However, when $\mathrm{x}$ has multiple targets, the nature of effective interactions from $\mathrm{x}$ to its targets becomes undetermined (see Figure 4C and example in Figure S14).

As an illustrative example of the graphical rules in Figure 4A-C, we show the effective interaction 
graph of a two-stage repression cascade in Figure 4D. Both $u$ and $\mathrm{x}_{1}$ are repressors with only one target. Therefore, applying the rule in Figure 4B, we have $\mathrm{u} \dashv \mathrm{x}_{1}$, and $\mathrm{x}_{1} \dashv \mathrm{x}_{2}$. Since $\mathrm{x}_{2}$ is not a target of repressor $\mathrm{u}$, applying the rule in Figure $4 \mathrm{~A}$, we have $\mathrm{u} \rightarrow \mathrm{x}_{2}$. By the same token, $\mathrm{x}_{1}$ is effectively activating itself. Since $\mathrm{x}_{2}$ does not transcriptionally activate or repress a target, there is no effective interaction initiated from $\mathrm{x}_{2}$. The resultant effective interaction graph in Figure 4D is a type 4 coherent feed-forward loop [29], whose dose response curve is monotonically increasing regardless of parameters (SI Section B7). Since a two-stage repression cascade and a two-stage activation cascade have interchangeable functions [29], our model suggests that a repression cascade is a more preferable topology in terms of robustness to resource competition.

\section{Discussion}

Gene expression relies on transcriptional and translational resources, chiefly RNAPs and ribosomes. As all genes in a circuit compete for these limited resources, unintended non-regulatory interactions among genes arise. These interactions can dramatically change the intended behavior of a genetic circuit. In this paper, through a combined modeling and experimental study, we have characterized the extent to which resource competition affects a genetic circuit's behavior. We have incorporated resource competition into standard Hill-function models through resource demand coefficients, which can be readily tuned by key circuit parameters such as RBS strength and DNA copy number. These coefficients dictate the strengths of non-regulatory interactions and can be effectively used to guide the design of a genetic circuit toward the intended behavior. Our mathematical model further provides a simple graphical tool to identify the nature of non-regulatory interactions (i.e. activation vs. repression) and to create the effective interaction graph of the circuit. Under the guidance of the model, we created a library of genetic activation cascades and demonstrated that, by tuning the resource demand coefficients of the cascade's nodes, the strengths of non-regulatory interactions can be predictably controlled and intended cascade's response can be restored.

Previous theoretical studies have analyzed how competition for common resources affects gene expression. Using a stochastic model [13], Mather et al. found a strong anti-correlation of the proteins produced by ribosome-competing mRNAs. Rondelez [28] developed a general model to describe substrates competing for a limited pool of enzymes. De Vos et al. [11] analyzed the response of network flux toward changes in total competitors and common targets. More recently, Raveh et al. [14] developed a ribosome flow model to capture simultaneous mRNA translation and competition for a common pool of ribosomes. In [12], Gyorgy et al. developed a mechanistic resource competition model that gives rise to "isocost lines" describing tradeoffs in gene expression, which were experimentally validated. All these models, with the exception of [28], are restricted to circuits without regulatory links among competing nodes. In contrast, our general model explicitly accounts for regulatory interactions among nodes and reproduce the "isocost lines" of [12] as a special case (see SI Section B4.2). Furthermore, differently from [28], our model couples the resources' enzymatic reactions with the slower gene expression reactions to obtain a model for resource-limited genetic circuits.

Previous experimental studies have provided evidence that transcriptional and translational resources may be limited in the cell by showing that DNA copy number, mRNA concentration, and protein concentration do not always linearly correlate with each other [24, 26]. Accordingly, there has been extensive experimental evidence that synthetic genes' over-expression inhibits host cell growth $[7,35,8,9]$. However, the effects of competition for shared resources on genetic circuits have only been recently addressed, mostly focusing on the single-gene effects as opposed to investigating the emergent effects on network level $[10,12,22,36]$. In this paper, we have theoretically predicted and experimentally demonstrated that significant network-level effects arise due to non-regulatory interactions dictated by resource competition. These interactions need to be accounted for in circuit design and optimization. Accordingly, we have provided a model-based approach to guide genetic circuit design to mitigate the effects of unintended interactions.

As a form of host-circuit interaction, previous studies have shown that change in host cell growth rate affects gene expression $[7,8,9,37]$. In our experiments of CAS $0.3 / 30$ and CAS 0.3/60, we observed very modest change in growth rates for all AHL concentrations. In experiments of CAS 1/60 and CAS 1/30, appreciable decrease in growth rates were observed at high AHL concentrations 
(see Figure S6). While growth rate change could potentially affect GFP expression, it is insufficient to explain the biphasic or monotonically decreasing dose response curves in Figure 3. Firstly, unintended effects of resource competition can already be observed in the dose response curve of CAS 0.3/60 for all AHL concentrations and those of CAS 1/30 and CAS 1/60 for low AHL concentrations. In these situations, growth rates were essentially unchanged (see Figure 3 and Figure S6). Secondly, according to the experimental results and the mathematical model in [9], expression of an activated gene increases as growth rate decreases. Therefore, in principle, slower growth rate at high AHL concentrations should result in higher GFP concentration, rather than in lower GFP concentration, as we observed in Figure 3 (see SI Section A4 for more details).

As circuits grow in size and complexity, a "resource-aware" design approach needs to be adopted by synthetic biologists. While resource competition can be exploited in certain situations to our advantage $[38,39,40]$, its global and nonlinear features largely hamper our capability to carry out predictive design. To alleviate the effects of resource competition, metabolic engineers down-regulate undesired gene expression to re-direct resources to the pathway of interest, thus increasing its yield $[41,42]$. Similarly, in a genetic circuit, we can tune the resource demand coefficients of nodes by selecting appropriate RBS and DNA copy numbers to diminish the resource demand by certain nodes and hence make more resource available to other nodes. This tuning should be performed by keeping in mind other design specifications that the circuit may have, such as maximal output or sensitivity of the dose response curve [1]. A simulation example of how to relate easily tunable parameters, such as RBS strength and DNA copy number, to circuit's output is given in SI Section B3.3 for the genetic activation cascade. At the higher abstraction level of circuit topology, our model helps to identify topologies whose behavior is less sensitive to the effects of non-regulatory interactions. We provided an example of this with the two-stage activation and repression cascades. While the dose response curve of the former can be completely reshaped by non-regulatory interactions due to resource competition, the dose response curve of the latter maintains its monotonically increasing behavior independent of resource competition.

Characterization of resource competition has deep implications in the field of systems biology, in which a major task is to reconstruct networks from data. In this case, it is critical to distinguish direct regulatory interactions from indirect ones [43], which may arise from non-regulatory interactions due to resource competition. In this sense, our model may provide deeper insights to guide the identification of natural networks from perturbation data.

\section{Associated content}

Methods and materials, detailed experimental data and mathematical models are described in Supporting Information.

\section{Acknowledgements}

We thank Fiona Chandra and Andras Gyorgy for helpful discussions and suggestions. YQ and HHH were supported in part by AFOSR grant FA9550-14-1-0060 and ONR award N000141310074. JJ was supported by BBSRC grant BB/M009769/1.

\section{References}

[1] Jennifer A N Brophy and Christopher A Voigt. Principles of genetic circuit design. Nat. Methods, 11(5):508-520, 2014.

[2] Stefano Cardinale and Adam Paul Arkin. Contextualizing context for synthetic biology- identifying causes of failure of synthetic biological systems. Biotechnol. J., 7:856-866, 2012.

[3] Domitilla Del Vecchio. Modularity, context-dependence, and insulation in engineered biological circuits. Trends Biotechnol., 33(2):111-119, 2015.

[4] Domitilla Del Vecchio, Alexander J Ninfa, and Eduardo D Sontag. Modular cell biology: retroactivity and insulation. Mol. Syst. Biol., 4:161, 2008. 
[5] Shridhar Jayanthi, Kayzad Soli Nilgiriwala, and Domitilla Del Vecchio. Retroactivity controls the temporal dynamics of gene transcription. ACS Synthetic Biology, 2(8):431-441, 2013.

[6] Deepak Mishra, Phillip M Rivera, Allen Lin, Domitilla Del Vecchio, and Ron Weiss. A load driver device for engineering modularity in biological networks. Nat. Biotechnol., 32:1268-1275, 2014 .

[7] Francesca Ceroni, Rhys Algar, Guy-Bart Stan, and Tom Ellis. Quantifying cellular capacity identifies gene expression designs with reduced burden. Nat. Methods, 12(5):415-422, 2015.

[8] Thomas E Gorochowski, Irem Avcilar-kucukgoze, Roel A L Bovenberg, Johannes A Roubos, and Zoya Ignatova. A Minimal Model of Ribosome Allocation Dynamics Captures Trade- offs in Expression between Endogenous and Synthetic Genes. ACS Synth. Biol., 5(7):710-720, 2016.

[9] Stefan Klumpp, Zhongge Zhang, and Terence Hwa. Growth-rate dependent global effect on gene expression in bacteria. Cell, 139:1366-1375, 2009.

[10] M. Carbonell-Ballestero, E. Garcia-Ramallo, R. Montañez, C. Rodriguez-Caso, and J. Macía. Dealing with the genetic load in bacterial synthetic biology circuits: convergences with the ohm's law. Nucleic Acids Res., 44(1):496-507, 2015.

[11] Dirk De Vos, Frank J. Bruggeman, Hans V. Westerhoff, and Barbara M. Bakker. How molecular competition influences fluxes in gene expression networks. PLoS One, 6(12):e28494, 2011.

[12] Andras Gyorgy, José I. Jiménez, John Yazbek, Hsin-Ho Huang, Hattie Chung, Ron Weiss, and Domitilla Del Vecchio. Isocost lines describe the cellular economy of gene circuits. Biophys. J., 109(3):639-646, 2015.

[13] William H. Mather, Jeff Hasty, Lev S. Tsimring, and Ruth J. Williams. Translation cross talk in gene networks. Biophys. J., 104:2564-2572, 2013.

[14] Alon Raveh, Michael Margaliot, Eduardo D Sontag, and Tamir Tuller. A model for competition for ribosomes in the cell. J. R. Soc. Interface, 13:20151062, 2015.

[15] Caleb J Bashor and James J Collins. Insulating gene circuits from context by RNA processing. Nature Biotechnology, 30:1061-1062, 2012.

[16] Chunbo Lou, Brynne Stanton, Ying-Ja Chen, Brian Munsky, and Christopher A. Voigt. Ribozyme-based insulator parts buffer synthetic circuits from genetic context. Nature Biotechnology, 30(11):1137-1142, 2012.

[17] Vivek K Mutalik, Joao C Guimaraes, Guillaume Cambray, Colin Lam, Marc Juul Christoffersen, Quynh-Anh Mai, Andrew B Tran, Morgan Paull, Jay D Keasling, Adam P Arkin, and Drew Endy. Precise and reliable gene expression via standard transcription and translation initiation elements. Nature Methods, 10:354-360, 2013.

[18] Kayzad Soli Nilgiriwala, José I. Jiménez, Phillip Michael Rivera, and Domitilla Del Vecchio. Synthetic tunable amplifying buffer circuit in E. coli. ACS Synth. Biol., 4(5):577-584, 2015.

[19] Wenlin An and Jason W. Chin. Synthesis of orthogonal transcription-translation networks. Proc. Natl. Acad. Sci. U. S. A., 106(21):8477-8482, 2009.

[20] Bartholomew Canton. Engineering the interface between cellular chassis and synthetic biological systems. PhD thesis, Massachusetts Institute of Technology, 2008.

[21] Manish Kushwaha and Howard M Salis. A portable expression resource for engineering crossspecies genetic circuits and pathways. Nature communications, 6:7832, 2015.

[22] Thomas H Segall-Shapiro, Adam J Meyer, Andrew D Ellington, Eduardo D Sontag, and Christopher A Voigt. A "resource allocator" for transcription based on a highly fragmented T7 RNA polymerase. Mol. Syst. Biol., 10:742, 2014. 
[23] Hans Bremer and Patrick P. Dennis. Modulation of chemical composition and other parameters of the cell by growth rate. In Frederick C. Neidhardt, editor, Escherichia coli and Salmonella: Cellular and Molecular Biology. ASM Press, 1996.

[24] Gordon Churchward, Hans Bremer, and RY Young. Transcription in bacteria at different DNA concentrations. J. Bacteriol., 150(2):572-581, 1982.

[25] Daniel M. Stoebel, Antony M. Dean, and Daniel E. Dykhuizen. The cost of expression of escherichia coli lac operon proteins is in the process, not in the products. Genetics, 178(3):16531660, 2008.

[26] Jesper Vind, Michael A. Sørensen, Michael D. Rasmussen, and Steen Pedersen. Synthesis of proteins in Escherichia coli is limited by the concentration of free ribosomes: Expression from reporter gene does not always reflect functional mRNA levels. J. Mol. Biol., 231:678-688, 1993.

[27] Dan Siegal-gaskins, Zoltan A Tuza, Jongmin Kim, Vincent Noireaux, and Richard M Murray. Gene circuit performance characterization and resource usage in a cell- free 'breadboard'. $A C S$ Synth. Biol., 3:416-425, 2014.

[28] Yannick Rondelez. Competition for catalytic resources alters biological network dynamics. Phys. Rev. Lett., 108(1):018102, 2012.

[29] Uri Alon. An Introduction to Systems Biology: Design Principles of Biological Circuits. Chapman \& Hall/CRC Press, 2006.

[30] Domitilla Del Vecchio and Richard M. Murray. Biomolecular Feedback Systems. Princeton University Press, Princeton, 2014.

[31] Stefan Klumpp. Mechanisms and economy of molecular machines. Phys. Scr., T151:014066, 2012.

[32] Shai Kaplan, Anat Bren, Erez Dekel, and Uri Alon. The incoherent feed-forward loop can generate non-monotonic input functions for genes. Mol. Syst. Biol., 4:203, 2008.

[33] Dongsan Kim, Yung-Keun Kwon, and Kwang-Hyun Cho. The biphasic behavior of incoherent feed-forward loops in biomolecular regulatory networks. Bioessays, 30(11-12):1204-1211, 2008.

[34] Hwi Park Hoo, Ki Lim Woon, and Hae J. Shin. In vitro binding of purified NahR regulatory protein with promoter Psal. Biochimica et Biophysica Acta - General Subjects, 1725(2):247-255, 2005.

[35] Henjiang Dong, Lars Nilsson, and Charles G. Kurland. Gratuitous overexpression of gene in Escherichia coli leads to growth inhibition and ribosome destruction. J. Bacteriol., 177(6):14971504, 1995.

[36] Jeffrey J Tabor, Travis S Bayer, Zachary B Simpson, Matthew Levy, and Andrew D Ellington. Engineering Stochasticity in Gene Expression. Mol. Biosyst., 4(7):754-761, 2008.

[37] Cheemeng Tan, Philippe Marguet, and Lingchong You. Emergent bistability by a growthmodulating positive feedback circuit. Nat. Chem. Biol., 5(11):842-848, 2009.

[38] Anthony J. Genot, Teruo Fujii, and Yannick Rondelez. Computing with competition in biochemical networks. Phys. Rev. Lett., 109(20):208102, 2012.

[39] Jongmin Kim, John Hopfield, and Erik Winfree. Neural Network Computation by In Vitro Transcriptional Circuits. In L K Saul, Y Weiss, and L Bottou, editors, Advances in Neural Information Processing Systems Vol.17, pages 681-688. MIT Press, 2004.

[40] Arthur Prindle, Jangir Selimkhanov, Howard Li, Ivan Razinkov, Lev S Tsimring, and Jeff Hasty. Rapid and tunable post-translational coupling of genetic circuits. Nature, 508(7496):387-391, 2014. 
[41] K L Jones, S W Kim, and J D Keasling. Low-copy plasmids can perform as well as or better than high-copy plasmids for metabolic engineering of bacteria. Metab. Eng., 2(4):328-338, 2000.

[42] Kevin V. Solomon and Kristala L J Prather. The zero-sum game of pathway optimization: Emerging paradigms for tuning gene expression. Biotechnol. J., 6(9):1064-1070, 2011.

[43] Taek Kang, Richard Moore, Yi Li, Eduardo Sontag, and Leonidas Bleris. Discriminating direct and indirect connectivities in biological networks. Proc. Natl. Acad. Sci., 112(41):12893-12898, 2015 . 